\title{
Pathophysiology of internal hemorrhoids
}

\author{
Nikolaos Margetis
}

University of Athens; Athens Euroclinic, Athens, Greece

Abstract

Hemorrhoidal disease is a fairly common and debilitating clinical entity. Despite centuries' of attempts to shed light on its pathophysiology, to cure those affected and to improve sufferers' quality of life, many aspects of the disease remain elusive. Individual beliefs and historical legends, accompanied by undocumented theories, have established and perpetuated the confusion regarding the mechanisms leading to the development of the disease and the rules governing its treatment. Hemorrhoids are classified as internal or external and are viewed as a disease when they become symptomatic. Returning to basic medical sciences, this mini-review focuses on internal hemorrhoids and aims to define the histology and anatomy of the normal and abnormal internal hemorrhoidal plexus and to encourage clinicians to comprehend the pathophysiology of the disease. If doctors can understand the pathophysiology of hemorrhoidal disease, they will be able to clarify the nature of the associated symptoms and complications and to make the correct therapeutic decision.

Keywords Internal hemorrhoids, anal cushions, anal nodules, prolapse, sliding anal canal

Ann Gastroenterol 2019; 32 (2): 1-9

\section{Introduction}

Hemorrhoidal disease has been recorded through centuries of history. Primitive references are found in the Old Testament and in Egyptian, Babylonian and Greek written sources [1-3]. Hippocrates approached the pathophysiology and the treatment of the disease in his treatise "On Hemorrhoids" [3]. Many physicians have tried to detect and describe the precise pathophysiological events of symptomatic disease [2-4]. Many surgeons have tried to derive conclusions based on operative findings [2,3,5-7]. Despite numerous efforts, the true etiopathogenesis of hemorrhoidal disease still remains elusive [8-9].

Pathophysiology is not the sole aspect of hemorrhoids to lack full elucidation. Many other factors need more investigation: for instance, the epidemiology of the disease [10], the optimal therapy for every single stage of hemorrhoids [8], and an explanation for the transition of asymptomatic hemorrhoids to symptomatic [11]. Moreover, a detailed search in the literature regarding the anatomy and histology of hemorrhoidal tissue and the relationship between hemorrhoids and the portal

Endoscopy Unit, Athens Euroclinic, Greece

Conflict of Interest: None

Correspondence to: Nikolaos Margetis, 46 Gennimata Street, 15238

Halandri, Athens, Greece, e-mail: nmargetis@yahoo.gr

Received 2 October 2018; accepted 11 December 2018; published online 23 January 2019

DOI: https://doi.org/10.20524/aog.2019.0355 venous system returns conflicting information $[7,10,12,13]$. Additionally, real life has proven that significant parts of the accepted wisdom regarding hemorrhoids are in fact clinical misunderstandings and personal beliefs passed on from one unsuspecting generation to the next. The purpose of this manuscript is to dispel these legends and to provide a welldocumented account of the etiopathogenesis of internal hemorrhoidal disease.

\section{Normal anatomy and histology of hemorrhoids}

Hemorrhoids are normal structures of the human body [9-11]. Internal hemorrhoids arise from the internal hemorrhoidal plexus, while external hemorrhoids arise from the external plexus. The anatomical boundary that divides the internal from the external hemorrhoidal plexus is the dentate line [8-10]. The normal internal hemorrhoidal plexus consists of 3 soft engorgements, referred to as anal cushions or "hemorrhoids" [14,15]. Consequently, the term "internal hemorrhoids" does not signify a state of disease if viewed in its strict literal definition. However, in clinical practice the term "internal hemorrhoids" is used to describe solely the disease resulting from the abnormal enlargement of anal cushions, i.e., their transformation to anal nodules $[10,14,15]$. More precisely, this definition is limited to symptomatic hemorrhoidal disease: i.e., anal cushions are named "hemorrhoids" when they bleed and/or prolapse [16].

The internal hemorrhoidal plexus is placed submucosally above the dentate line and below the anorectal ring [17]. 
It extends from the upper border of the anatomical anal canal to the upper border of the surgical anal canal. It is located, therefore, outside the anatomical anal canal. It is covered by transitional columnar epithelium and originates embryologically by the cloacal part of the anal canal, which contains both ectodermal and endodermal elements [14]. This epithelium is approximately $1 \mathrm{~cm}$ long [14] and, as anatomically authentic rectal epithelium, it secretes mucus [10] and is not innervated by visceral pain fibers [16].

\section{The vascular part of the anal cushion}

The hemorrhoidal tissue located between the mucosa of the surgical anal canal and the internal sphincter is not uniform in its appearance. The hemorrhoidal vasculature is normally condensed in the form of anal cushions; these cushions represent well-shaped, deep-purple, hemispherical masses, which protrude towards the lumen of the upper surgical anal canal $[14,15]$. Three main anal cushions are easily recognized in the usual surgical anal canal $[15,18]$. Despite being tissues that are predominantly vascular in origin, anal cushions also contain a significant non-vascular portion. The internal hemorrhoidal plexus consists of arterioles, venules and direct communications between them (arteriolar-venular functional anastomoses). The presence of vessels inside cushions contributes to the scarlet color of the surgical anal canal [14].

The internal hemorrhoidal plexus receives blood from the superior (SRA) and the middle (MRA) rectal arteries. In the majority of cases these arteries form a plexus just behind the rectum. This plexus, absolutely distinct from the internal hemorrhoidal plexus, commonly provides 3 principal terminal branches, which penetrate the rectal wall, ultimately terminating submucosally in the anus above the dentate line in 3 positions: the left lateral, the right anterior and the right posterior position.

Despite the conflicting reports in reference textbooks regarding veins emerging from anal cushions $[5,7,10,12,14,18]$, venous drainage from the internal hemorrhoidal plexus is currently widely accepted to be directed to all 3 rectal veins [14], which communicate with each other $[5,7,10]$. The superior (SRV) and middle (MRV) rectal veins are commonly the predominant venous branches of the internal hemorrhoid cushions $[7,10]$. The SRV drains blood to the inferior mesenteric vein, which belongs to the portal venous circulation, while the MRV and the inferior rectal veins are tributaries of the systemic circulation. Consequently, blood from anal cushions is drained through both circulations, systemic and portal.

Inside cushions, blood moves directly from arterioles to venules, via a plethora of arteriolar-venular anastomoses. Most arteriolar-venular anastomoses lack muscular wall; therefore, they are considered as sinusoids $[15,16]$. The cluster of sinusoids forms a spongy capillary network that resembles penile erectile tissue, since sinusoids consist of numerous, dilated, uneven vascular spaces covered by endothelium [7]. Sinusoidal hemorrhoidal tissue is an imaginary 3-way crossroad, which initially receives arterial blood from the SRA and MRA, oxygenates the non-vascular part of the cushion, and ultimately emits venous blood to the tiny root venules of the SRV and MRV. In this way, blood from the arterial, systemic venous and portal venous circulations is mixed inside the sinusoids (Fig. 1).

Why is the hemorrhoidal vasculature concentrated into 3 distinct anal cushions? Several theories have been developed, none of which fully explains their formation [13-17]. The preferential arterial supply into specific locations of the upper anus favors the development of sinusoidal plexus in these locations almost exclusively. Indeed, the position of the anal cushions corresponds directly to the location of the terminal principal arterial branches in the submucosal tissue: left lateral (3 o'clock), right anterior (11 oclock) and right posterior (7 oclock) $[14,18]$. An alternative explanation is that normal anal cushions are not real protrusions; they rather represent the unavoidable creases appearing during the closure of the anal canal and disappearing when the anal canal widens during defecation. The aim of these folds is to seal the anal canal in the resting state [17].

\section{The crucial role of the non-vascular part of the anal cushion}

The non-vascular part of the cushion consists of the transitional epithelium, connective tissue, elastic and collagenous, and the muscle of Treitz $[8,16]$. Treitz's muscle tightly maintains the cushions in their normal position; its deterioration is considered one of the most important pathogenetic factors in the formation of hemorrhoids. Treitz's muscle consists of 2 distinct parts: the anal submucosal muscle, whose fibers subside submucosally between the sinusoids, fixes the cushions to the "floor" of the hemorrhoids (i.e., to the internal anal sphincter), while the mucosal suspensory ligament (Park's ligament) penetrates the internal sphincter and fixes the sinusoids to the conjoined longitudinal muscle [17].

Although the conjoined longitudinal muscle is not an anatomical part of the anal cushion, it is just as important as Treitz's muscle for the prevention of hemorrhoidal disease. It appears to function like a backbone, which supports the internal and the external sphincter and fixes the anorectum to the pelvis. It is located just beneath the internal sphincter and serves as the constant platform where the hemorrhoidal tissue is fixed via Park's ligament [17]. Moreover, its fibers form a loop at the distal end of the internal sphincter. This is the reason why some investigators claim that conjoined longitudinal muscle continues above the internal anal sphincter to form the anal submucosal muscle [19] (Fig. 1).

The firm fixation of normal anal cushions above the dentate line, the maintenance of their usual size and the prevention of their prolapse is ensured by 4 anatomical safety features: first, the integrity of the accompanying connective tissue found between the sinusoids, which supports sinusoids and fixes them to the internal sphincter [20]; second, the anatomical entirety of Treitz's muscle and of the conjoined longitudinal muscle [15,17]; third, the sphincter-like structure present in the terminal 


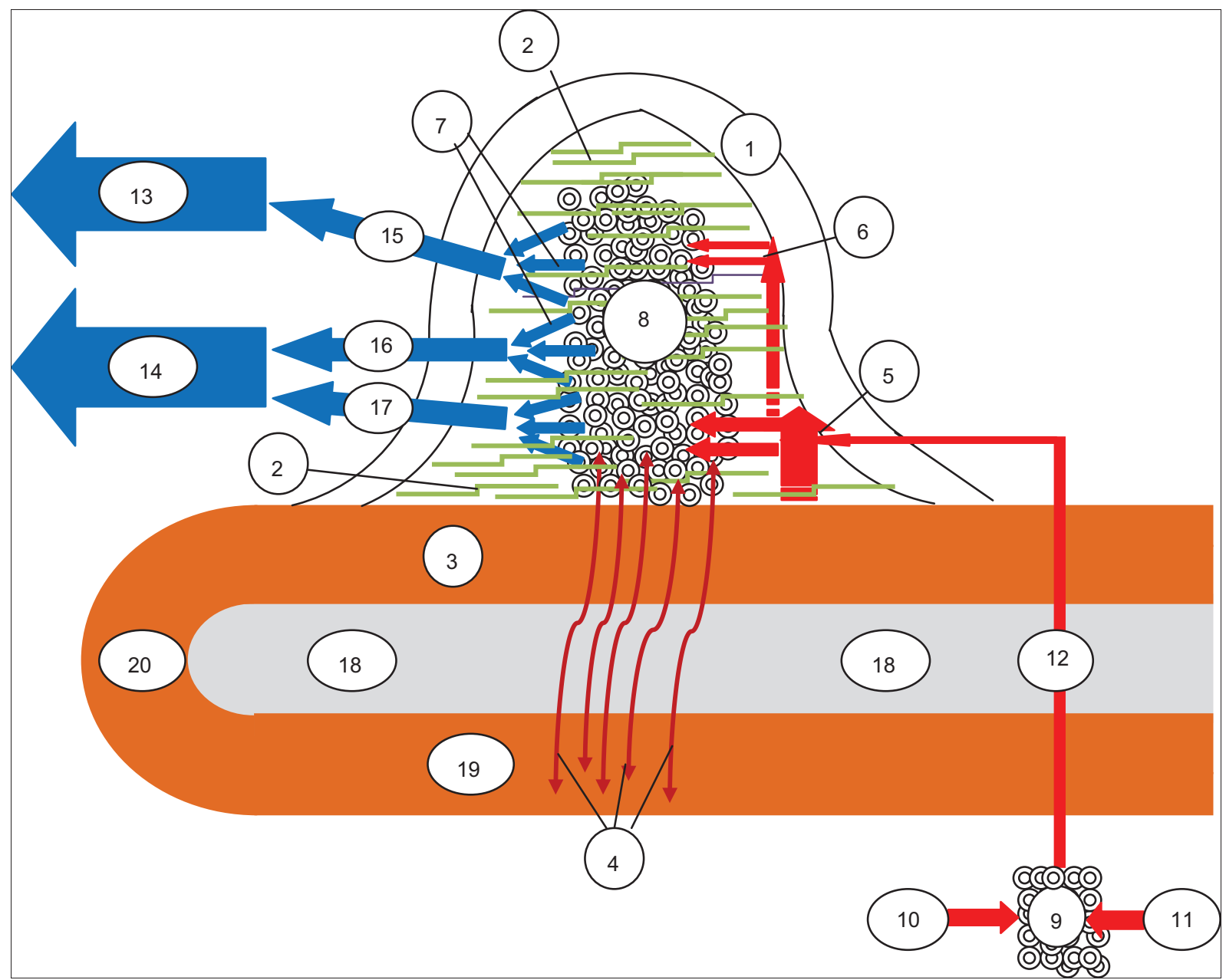

Figure 1 A proposed schematic representation of the anatomy of the anal cushion. Anal cushion consists of a non-vascular part (the transitional epithelium - 1, the submucosal connective tissue -2 and the muscle of Treitz, which has 2 elements, the anal submucosal muscle - 3 and the mucosal suspensory ligament or Park's ligament - 4) and a vascular part (the small arterioles -5 , the tiny arterioles -6 , the tiny venules -7 and the direct arteriolar-venular anastomoses between arterioles and venules, that form a subcutaneous spongy network of capillaries, the sinusoids - 8). Arterial blood from the retrorectal plexus - 9 (formed by the SRA -10 and MRA -11) supplies the sinusoidal plexus via 3 terminal arterial branches (here one of them is shown - 12). Before turning into sinusoids - 8, the terminal branch subdivides into small - 5 and tiny - 6 arterioles in the lamina propria. These small and tiny arterioles are the source of bleeding, when the anal cushion is transformed to an anal nodule. Sinusoid drainage is accomplished via tiny venules - 7, which direct venous blood both to the portal circulation - 13 (through the SRV -15) and to the systemic circulation - 14 (mainly through the MRV-16 and to a lesser extent through the IRV -17). The cluster of sinusoids is tightly fixed to the inner anal sphincter -18 by the anal submucosal muscle -3 and by the submucosal connective tissue -2 (which extends between the sinusoidal anastomoses) and to the conjoined longitudinal muscle -19 by the mucosal suspensory ligament -4 . According to several researchers, anal submucosal muscle -3 is thought to represent the continuity of the conjoined longitudinal muscle -19; the latter is believed to form a loop -20 at the lower end of the internal anal sphincter (based on references 5,7,8,10,12-20)

$I R V$, inferior rectal vein; $M R A$, middle rectal artery; $M R V$, middle rectal vein; SRA, superior rectal artery; SRV, superior rectal vein

arterioles; and fourth, the fairly small caliber of the terminal branches of the supplying arteries [13,20]. The last 2 anatomical features also prevent the hyperperfusion of sinusoids [13]. If the aforementioned structures fail anatomically or functionally, hemorrhoidal disease emerges $[8,10,13,15,17,20]$.

These anatomical relationships bear physiological importance and contribute to the uneventful function of the anal region. Anal continence is ensured by the interaction between the hemorrhoidal tissue and the internal anal sphincter. The internal anal sphincter is responsible for $60-80 \%$ of normal anal pressure. Nevertheless, the internal sphincter leaves a gap of 7-8 $\mathrm{mm}$, closed as long as the anal cushions demonstrate anatomical and functional integrity [15]. Therefore, the $15-20 \%$ contribution of hemorrhoids to anal continence is crucial [21]. During the resting state, the increased pressure of the internal anal sphincter impedes the venous drainage of anal cushions, enhancing their congestion and contributing to their notable size. This volume-occupying effect seals the anal canal and continence is achieved $[10,15]$. Just before defecation, hemorrhoidal tissue contributes to the sensing of a sample of intrarectal contents (liquid, gas, stool), as it has sensory innervations [22]. 
During defecation, the hemorrhoids and internal anal sphincter aid one another: the lowered pressure of the relaxed internal anal sphincter permits blood to drain from the anal cushions to the SRV and MRV, helping to reduce them in size. Two more mechanisms contribute to the decrease in anal cushion size: the direct dilation of the anal canal by the descending stool and the contraction of the anal cushions by the submucosal ligament of Treitz's muscle. At the same time, the anal cushions slide down, forming anal lips [15], which protect the underlying internal anal sphincter [23]. As the anal cushions return upwards to their usual position after defecation, they rapidly fill with blood until they reach their usual size. In this way, fecal soiling is prevented $[14,15,17]$.

\section{The pathologic basis of hemorrhoidal disease}

The pathophysiology of internal hemorrhoids is still not fully understood. Many functional, histopathological and anatomical findings have been accumulated during the last decades, but the relation between them remains obscure. In the majority of cases, we do not know whether they are primary or secondary. Despite the plethora of candidates for the initial steps, we are not aware which is the most prevalent. Every single hemorrhoid patient demonstrates a different combination of starting points and distinct pathophysiological events, connected in a personalized order. As the proposed algorithm in Fig. 2 illustrates, the pathophysiology is complicated and comprises many vicious cycles.

Four theories have been developed regarding the pathophysiology of hemorrhoids. First, the varicose vein theory has been shown to be faulty [15], as it is now globally accepted that hemorrhoids are not varicosities [24]. Theories involving vascular hyperplasia (the theory that hemorrhoids resemble penile erectile tissue) and internal anal sphincter hypertonia hold part of the truth, but they cannot be totally accepted $[10,13,15,17]$. The globally accepted theory is that of the sliding anal canal, or cushion theory, which postulates the abnormal slippage of cushions through the anal canal as the major pathophysiological event [15].

A look at Fig. 2 makes it clear that many events are believed to initiate the disease. Regardless of the initiation points, 4 core pathophysiological events are universally recognized: first, the sliding process of anal cushions; second, the deterioration of the connective tissue of the cushion; third, the reduction of venous return from sinusoids to the SRV and MRV during defecation; and fourth, the stagnation of blood inside the dilated plexus. These crucial events are highlighted in red in Fig. 2.

A chronic rise in intraabdominal pressure, in combination with the absence of valves within rectal veins, can limit venous drainage from sinusoids during defecation, resulting in abnormal dilatation of the arteriolar-venular anastomoses of the internal hemorrhoidal plexus $[10,25]$. Human erect position, pregnancy, obesity, ascites [25], straining with defecation $[16,22]$, excessive time spent on the toilet [22], strenuous lifting [16] and strenuous exercise [24] may all cause an excessive increase in intraabdominal pressure. Furthermore, human civilization has adopted the sitting position during defecation, which does not permit the straightening of the anorectal angle (in contrast, this angle is eliminated if defecation is performed in the primitive squatting position), necessitating even greater effort and exaggerated intraabdominal pressure to overcome the relative obstacle met by the descending stool $[8,27]$. Nevertheless, these factors have not been studied in detail [8]. Pregnancy, for instance, was not associated with hemorrhoids in 2 recent studies [26,28], whereas an increased body mass index had only an ambiguous relationship with hemorrhoid development [26,28].

Although constipation (due to the absence of dietetic fibers, among other factors), has traditionally been considered an important risk factor for hemorrhoid development [10], recent studies doubt on this relationship $[29,30]$. Nonetheless, constipation increases intraabdominal pressure and directly impedes venous return, via the effect of hard stool in the rectal veins [30], while hard stool applies strong intra-anal forces to the anal cushions during defecation [13]. Moreover, prolonged defecation attempts in the constipated patient may lead to repeated and ineffective evacuation, which impedes the venous return to hemorrhoids even more.

Anal submucosal muscle firmly connects anal cushions to the internal sphincter. Park's ligament firmly connects anal cushions to conjoined longitudinal muscle. Since the anal cushions and internal sphincter move in opposite directions (the former slips down, the latter relaxes), both parts of Treitz's muscle are elongated and stretched during defecation. Repeated and successive cycles of elongation and shortening of Treitz's muscle following chronic strained defecations result initially in its relaxation and ultimately in its disintegration [15,31]. Treitz's muscle fibers may also disintegrate as a result of pelvic floor disorders [15] and with increasing age [25,32]. The final result is the persistence of the prolapsed anal cushions outside the anus after defecation. In the initial grades of hemorrhoidal disease ( $1^{\text {st }}$ and $2^{\text {nd }}$ degree), anal cushions reverse back into the anus without any effort from the patient. In advanced grades, the additional disintegration of conjoined longitudinal muscle results in their remaining permanently outside the anus, either manually reversible ( $3^{\text {rd }}$ degree $)$ or nonreversible $\left(4^{\text {th }}\right.$ degree $)[15,17]$.

Furthermore, relaxation of the supportive connective tissue enhances the permanent prolapse of anal cushions. First, functional or anatomical deterioration of Treitz's muscle per se disrupts the integrity of the connective tissue $[15,20]$. Second, abnormalities in the quality of collagen (loose collagen tissue is commonly found in Ehlers-Danlos syndrome [33] and in aging individuals [25]) lead to deterioration of the connective tissue. Indeed, an increase in matrix metalloproteinases (MMP)-2 and MMP-9 activity, known to degrade elastic fibers and to enhance tissue remodeling, is detected in patients with hemorrhoids [5,34]. Third, redundant connective tissue is generated as the overlying mucosa is stretched; fibrous tissue hypertrophy results in the loss of its elasticity and hence its ability to shorten after defecation [10,15]. Fourth, heredity confers a part in the disintegration of both Treitz's muscle and the supportive connective tissue; their deterioration with age may be familial [25]. Last, relaxation of the connective tissue is provoked by chronic or intermittent diarrhea; moreover, 


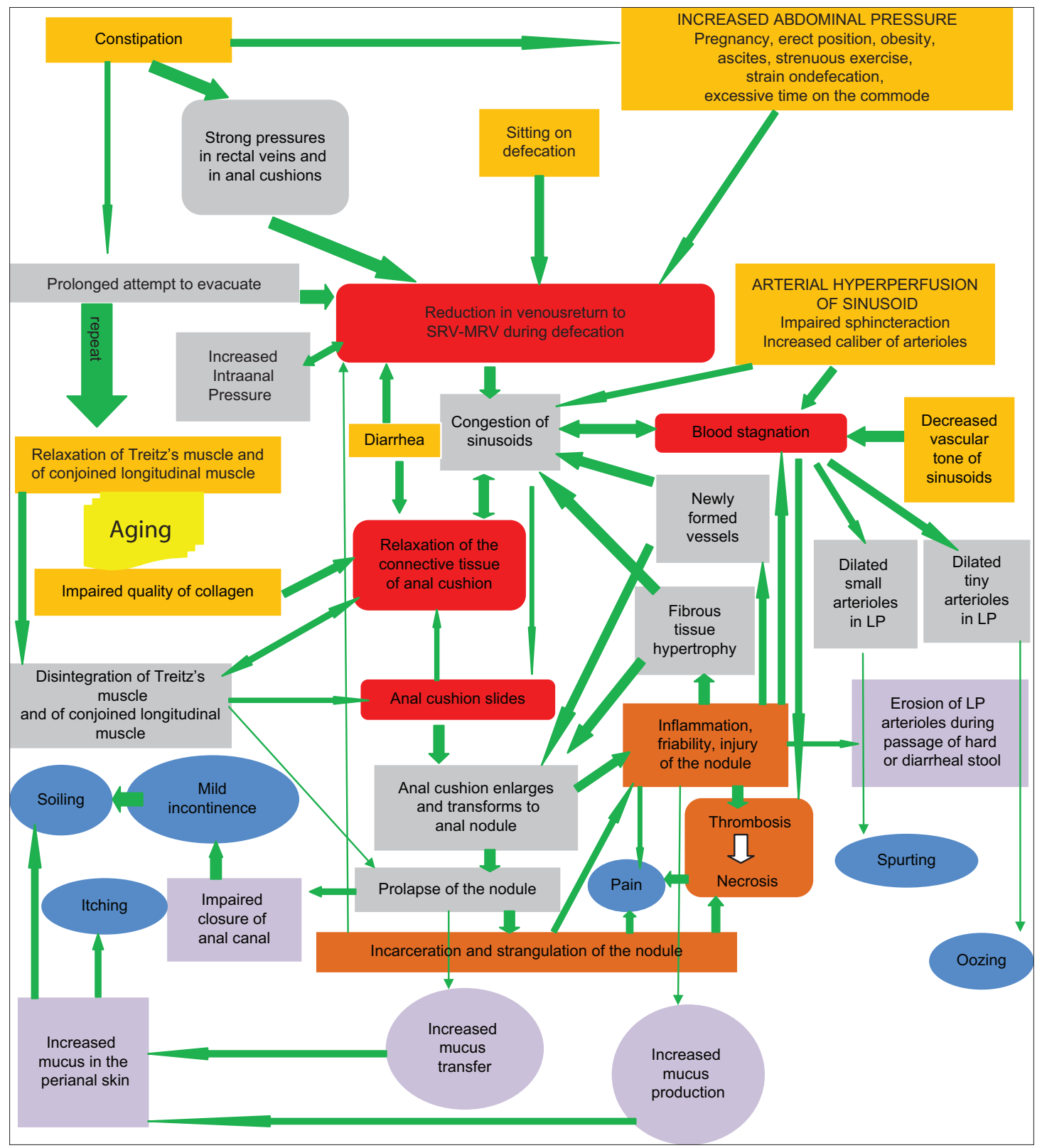

Figure 2 A suggested integrated algorithm summarizing contemporary thinking regarding the pathophysiology of internal hemorrhoids. Hemorrhoidal disease can arise from separate origins or from various combinations of them: these are the eight starting points, shown in the orange boxes (increased intraabdominal pressure, sitting on defecation, constipation, diarrhea, arterial hyperperfusion of the hemorrhoidal plexus, decreased vascular tone of sinusoids, impaired quality of collagen and relaxation of the supporting muscles of the internal hemorrhoidal plexus). These initiation points force the physiological and histological status of the internal hemorrhoidal plexus towards distinct, divergent, abnormal directions. Nonetheless, they finally converge on 4 central pathophysiological events that are almost invariably encountered in internal hemorrhoids. These events (sliding of the anal cushion, relaxation of the connective tissue of the cushion, reduction of venous return from sinusoids to SRV and MRV during defecation and stagnation of blood inside the dilated plexus) are illustrated in the red boxes. The bond between the starting points and the core pathophysiological events is strong and consists of a plethora of consecutive pathophysiological stages, indicated by the gray boxes, which are interconnected, interdependent and inter-fortified. The conceptual network of events leading to hemorrhoidal disease is complicated and has not been fully elucidated in every detail; nevertheless it is established and perpetuated with the assistance of many vicious cycles, either direct-for example between the increased intra-anal pressure and the reduction of venous return during defecation-or multi-step, as between the congestion of sinusoids and incarceration. It is noteworthy that this multimodal and multidirectional network is continuously auto-reinforced, as is implied by the universally green color of the arrows. This means that, according to this model, internal hemorrhoids offer only one option: to become worse over time. Ultimately, symptoms (shown in blue) occur. This happens when the order of events meets critical pathophysiological steps (shown in purple); the erosion of arterioles of the lamina propria of the anal nodule during passage of hard or diarrheal stool is typically responsible for the various forms of hemorrhoidal bleeding. Last, pain, a non-typical symptom of internal hemorrhoids, appears when complications (brown boxes) such as inflammation, incarceration, thrombosis and necrosis arise (based on references 4,7-11,13,15-17,19,2131,34,38,39)

$L P$, lamina propria; $M R V$, middle rectal vein; SRV, superior rectal vein 
chronic diarrhea exerts pressure on the draining venous system of sinusoids [29]. Indeed, diarrhea is currently believed to represent a stronger risk factor for hemorrhoids compared to constipation [35].

One central hallmark of hemorrhoidal disease is the congestion of sinusoids. This is primarily due to increased abdominal pressure and to the reduced venous return during defecation [15-18]. Moreover, arterial hyperperfusion of sinusoids (as a result of an impaired arteriolar sphincteric mechanism [36]) and the decreased vascular tone (because of the dominance of the endothelium-derived relaxing nitric oxide over the endotheliumderived vasoconstricting reactive oxygen radicals [37]) enhance the congestion of sinusoids. Additionally, the relaxed and hypertrophied connective tissue loses its capacity to support the vascular network; thus, sinusoids congest passively. Finally, increased internal anal sphincter activity $[17,38]$, either primary or secondary [23], impedes venous return, worsening congestion.

Apart from the above, cushion enlargement is amplified by fibrous tissue hypertrophy and by neovascularization. Newly formed vessels emerge when inflammation develops as hemorrhoids worsen $[10,13,40]$. Metalloproteinase overactivity leads to a breakdown of the capillary bed and permits the action of transforming growth factor beta (exhibits an angioproliferative effect) and of vascular endothelial growth factor (leads to sinusoidal hypertrophy) [13].

To make things more complex, 2 more vicious cycle are established, the first between sinusoidal congestion and the already loose and hypertrophied connective tissue of anal cushions $[4,10,11]$, and the second between the congestion of sinusoids and increased anal pressure due to a hypertonic internal anal sphincter [23].

Consequently, anal cushions become increasingly congested, enlarged and engorged and are transformed into abnormal anal cushions, anal nodules or "hemorrhoids". As they have also lost their muscular and fibrous fixation, they prolapse through the anal canal. The sliding down process of anal cushions is the most crucial pathophysiologic feature of the disease [15]. In this gradually worsening chronic process, the prolapsed nodules are trapped in the narrow anal canal where they are gradually strangulated and incarcerated, increasing anal pressure and venous obstruction and aggravating congestion. The hypercongested nodules then further exacerbate the incarceration and strangulation (vicious cycle) $[11,39]$.

Prolapse of the cushions can occur acutely as well. In acute prolapse, hemorrhoidal tissue is trapped abruptly by the sphincter mechanism outside the anus; this can ultimately lead to complete obstruction of venous return and strangulation [39]. As anal nodules prolapse through the anatomical anal canal, the tissue of the nodule becomes injured, inflamed and friable [10]. Indeed, a chronic serious inflammatory reaction involving both the vascular part (sinusoidal wall) and nonvascular part (supportive tissue) has been demonstrated in hemorrhoids $[13,40]$. The inflammatory process renders the arterioles of the lamina propria of the nodule vulnerable to erosion during defecation with resultant hemorrhage. This bleeding presents as oozing when tiny arterioles of the lamina propria are eroded, and as spurting when small arterioles are corroded [40].
The combination of sinusoidal congestion, strangulation [10] and chronic inflammatory process inside the nodule [40] results ultimately in blood stagnation inside sinusoids. Stagnation causes locally restrained hypercoagulability, which enhances clot formation and finally results in thrombosis. Thrombosis may eventually lead to ischemia, ulceration and necrosis of the surface of the nodule [13].

\section{Symptomatic hemorrhoidal disease}

Approximately 4 of 10 patients with hemorrhoids are symptomatic [28]. It is uncertain why hemorrhoids become symptomatic. They are believed to start producing symptoms when anal cushions start their sliding down process [5-7].

Bleeding is the commonest symptom of internal hemorrhoids [41]. In the vast majority of cases bleeding is painless, because of the absence of pain fibers [16]. Hemorrhage is due to congestion of the sinusoids, transferred backwards to the presinusoidal arterioles. Bleeding from hemorrhoids is therefore arterial in origin, hence bright red [7,10]. Since dilated arterioles are located in the lamina propria, just below the inflamed, injured and friable epithelium of the descending nodule, they are easily eroded during defecation by hard [25], dehydrated [22] or diarrheal [29,35] stool. As presinusoidal arterioles are usually tiny, oozing is the commonest type of bleeding; blood is present in the toilet paper exclusively and is typically associated with bowel movements $[16,23]$. When small arterioles are corroded, bright red blood will spurt [7]; in this case bright red blood drips into the toilet bowl during or immediately after evacuation $[11,16]$. Blood may rarely emerge from the newly-formed, hypertrophied arterioles, leading to serious bleeding, manifesting as blood spray around the toilet bowl [16]. In rare cases, blood from internal hemorrhoids may follow a cranial direction towards the rectum, and then return caudally to the anus, presenting as hematochezia. Bleeding is usually the first complaint to appear, before the hemorrhoids start to prolapse (first degree) $[11,42]$. In this initial stage, the dilatation of sinusoids is still in evolution, rendering them prone to bleeding, while the deterioration of the fibromuscular supporting tissue has just begun. Therefore, although hemorrhoidal cushions may be prominent on endoscopic evaluation, their prolapse is not obvious on strenuous clinical examination $[11,25]$.

The mucosa of the prolapsing nodule is rectal and mucusproducing [14]. The prolapsed mucosa, therefore, permits the passive transfer of a greater amount of mucus outside the anus. Moreover, the inflamed mucosa of the prolapsing nodule produces even more mucus [10]. The net result is the presence of increased mucus in the perianal skin, which causes perineal irritation, itching and soiling. Besides this, patients with prolapsed hemorrhoids lack the capability to sense the composition of rectal sample [22] and to seal the anus after defecation $[10,15,17]$. Consequently, they complain of mild fecal incontinence and of finding unknown material in their underclothes $[10,16]$. Prolapsed internal hemorrhoids are also manifested by rectal fullness and/or a feeling of incomplete 
evacuation, because of their space-occupying effect.

Internal hemorrhoids are not typically palpated on digital rectal examination, unless they are large enough or prolapsed $[9,13]$.

Pain is not the typical symptom, as already noted. Pain may be due to complicated internal hemorrhoids (thrombosis, ischemia, incarceration, strangulation) $[11,16]$. Nevertheless, thrombosis is a rare complication [10]. Consequently, when pain is the patient's prominent symptom and possible complications of internal hemorrhoids are not detected on clinical or endoscopic examination, the physician should search for an alternative diagnosis: for example, coexisting external hemorrhoids, perirectal abscess or anal fissure $[9,11,16]$.

Internal hemorrhoids may be found, clinically or endoscopically, in 3 distinct anatomical locations: first, above the dentate line, since they emerge and protrude from the mucosa of the upper anal canal [7-9]; second, below the dentate line, but remaining inside the anatomical anal canal; and third, outside the anal verge, in advanced cases.

\section{Medically valuable correlations}

1. One major goal of the conservative treatment of hemorrhoids is to normalize stool consistency; both diarrhea and constipation are clinically potent causes and triggering factors of symptoms in hemorrhoidal disease and their management is as invaluable as the treatment of hemorrhoids per se. The remaining elements of conservative therapy (e.g., avoiding of straining on defecation) have been proved to limit the prolapse of hemorrhoids $[43,44]$.

2. Increased anal pressure is very important in the pathophysiology of hemorrhoids and is a frequent clinical sign, manifested as anal spasm on rectal examination. Increased anal pressure is the rationale for using topical nitroglycerine $0.4 \%$ ointment in the treatment of pain due to internal hemorrhoids $[45,46]$. Intra-anal pressure returns to normal after hemorrhoidectomy [22].

3. The principle mode of action of flavonoids (especially the micronized ones, which are easily absorbed and act very shortly) is by increasing the impaired vascular tone [47]. Moreover, they are supposed to relieve congestion inside sinusoids (they reduce venous capacity and decrease capillary permeability [48]) to facilitate lymphatic drainage [49] and to exert anti-inflammatory effects [50]. Recent clinical trials have proven the effectiveness of treatment with flavonoids, both in chronic and in acute hemorrhoidal disease. In chronic disease, conservative therapy with micronized purified flavonoid fraction was found to be beneficial both in relieving hemorrhoidal symptoms and in preventing disease relapse in the majority of patients [51]. In acute hemorrhoidal disease, a mixture of flavonoids (diosmin, troxerutin and hesperidin) was shown to control symptoms efficiently and to manage both congestion and thrombosis of anal cushions [52].

4. Anti-inflammatory suppositories are used to improve the symptoms of hemorrhoids; the rationale of using them is that inflammation is the triggering factor of bleeding and contributes to pain and irritation of perianal skin $[10,11,40]$. Nonetheless, solid evidence to justify their efficacy in hemorrhoids is lacking [8-11].

5. Preparation-H, which contains $0.25 \%$ phenylephrine, petrolatum, light mineral oil and shark liver oil, relieves acute pain and bleeding on defecation by restoring the imbalance between vasoconstrictive and vasodilating agents inside sinusoids. Its vasopressor effect is attributed principally to phenylephrine, which acts mainly on the arterial branch of the hemorrhoidal plexus [53]. The clinical efficacy of this formulation in relieving hemorrhoidal symptoms has been proven in a recent prospective randomized open-label study [54].

\section{Surgically valuable correlations}

1. The principle of the "rubber-band ligation" technique is to band the mucosa and the submucosa of the anal cushion. Banding generates inflammation, with ultimate ischemia and necrosis of the banded part. Apart from removing the nodule, the final sequela of the procedure is the fibrosis generated: it fixes the remaining tissue in the underlying tissue of the surgical anal canal and corrects the prolapse $[16,21,41,55]$. It is considered the mainstay of therapy for hemorrhoids grade I and II $[10,22]$ and of selected patients with grade III $[10,56]$. Several trials have proven that this minimally invasive therapeutic procedure is efficient, reliable and safe for hemorrhoidal patients [56,57].

2. The modified (Ferguson) excision hemorrhoidectomy is currently the commonest type of hemorrhoidectomy. In this operation, both the prolapsed sinusoidal tissue and the hypertrophied connective tissue are removed, whereas the anoderm is preserved, the internal sphincter is protected and the sliding process, the fundamental pathophysiologic feature of hemorrhoids, is totally inverted [58-63].

3. The rationale of stapled hemorrhoidopexy is the fact that both the loose mucosa and the enlarged hemorrhoidal nodules above the dentate line contribute to the prolapse of internal hemorrhoids $[9,64]$. This operation is not a hemorrhoidectomy. Instead, a circular device excises a ring of redundant mucosa and fixes the hemorrhoidal plexus above the dentate line. During this operation, 3 critical nodes in the pathogenesis of hemorrhoids are targeted: first, prolapse is restored; second, the volume of the hemorrhoidal nodules is significantly reduced; and third, the vessels supplying the internal hemorrhoidal plexus are ligated, limiting the blood supply to the nodules [65].

4. Doppler-guided hemorrhoidal artery ligation offers a tailored, individualized therapy, since it locates the feeding terminal branch of the SRA that supplies each hemorrhoidal nodule [21], ultimately performing targeted suture ligation $[13,66]$. Consequently, the increased blood supply to the anal cushion is diminished, its engorgement is prevented, its size is decreased and it is ultimately firmly fixed to the floor of the upper surgical anal canal [67]. This alternative operational technique has been proven to relieve 
patients' symptoms, with only minor complications and rare relapses [68].

\section{Concluding remarks}

Overall, hemorrhoidal disease is a quite common and annoying disease, by definition symptomatic. It is imperative for clinicians who manage patients with internal hemorrhoids (gastroenterologists, surgeons, general practitioners) to be familiar with the normal and abnormal anatomy of hemorrhoids and to be capable of correlating their clinical findings with the subsequent pathophysiology. The pathophysiological explanation of clinical and/or endoscopic signs is an indispensable component of the roadmap to successful treatment of the hemorrhoidal patient.

\section{References}

1. Holley CJ. History of hemorrhoidal surgery. South Med J 1946;39:536-541.

2. Senagore AJ. Surgical management of hemorrhoids. J Gastrointest Surg 2002;6:295-298.

3. Ellesmore S, Windsor AC. Surgical history of haemorrhoids. In: Charles MV, (editor): Surgical treatment of haemorrhoids. Springer: London; 2002, pp. 1-4.

4. Hancock BD. Internal sphincter and the nature of haemorrhoids. Gut 1977;18:651-655.

5. Thomson WH. The nature of haemorrhoids. $\mathrm{Br} \quad J$ Surg 1975;62:542-552.

6. Gass OC, Adams J. Hemorrhoids; etiology and pathology. Am J Surg 1950;79:40-43.

7. Bernstein WC. What are hemorrhoids and what is their relationship to the portal venous system? Dis Colon Rectum 1983;26:829-834.

8. Sandler RS, Peery AF. Rethinking what we know about hemorrhoids. Clin Gastroenterol Hepatol 2019;17:8-15.

9. Mott T, Latimer K, Edwards C. Hemorrhoids: diagnosis and treatment options. Am Fam Physician 2018;97:172-179.

10. Ganz RA. The evaluation and treatment of hemorrhoids: a guide for the gastroenterologist. Clin Gastroenterol Hepatol 2013;6:593-603.

11. Jacobs D. Clinical practice. Hemorrhoids. N Engl J Med 2014;371:944-951.

12. Hull T. Disease of the anorectum. In: Feldman M, Friedman L, Brandt L (editors): Sleisenger and Fortran's Gastrointestinal and Liver Disease, $8^{\text {th }}$ edition, Vol II. Saunders Elsevier: Philadelphia; 2006, pp. 2833-2834.

13. Lohsiriwat V. Hemorrhoids: from basic pathophysiology to clinical management. World J Gastroenterol 2012;18:2009-2017.

14. Jose Marcio Neves Jorge, Angelita Habr-Gama. Anatomy and embryology of the colon, rectum, and anus. In Beck D, Roberts P, Saclarides T, Senagore A, Stamos M, Wexne S (editors): ASCRS Manual of Colon and Rectal Surgery, $2^{\text {nd }}$ edition. Springer: New York Dordrecht Heidelberg London; 2011, pp.1-22.

15. Hyung Kyu Yng. The pathophysiology of hemorrhoids. In: Hyung Kyu Yng (editor): Hemorrhoids. Springer-Verlag, Berlin Heidelberg; 2014, pp. 15-24.

16. Sun Z, Migaly J. Review of hemorrhoid disease: presentation and management. Clin Colon Rectal Surg 2016;29:22-29.

17. Hyung Kyu Yng. Anal anatomy. In: Hyung Kyu Yng (editor):
Hemorrhoids. Springer-Verlag, Berlin Heidelberg; 2014, pp. 5-14.

18. Blarucha AE, Wald A. Anorectal diseases. In: Tadataka Yamada (editor): Textbook of Gastroenterology, $5^{\text {th }}$ edition, Vol I. WileyBlackwell, West Sussex, 2009, pp. 1717-1718.

19. Lunniss PJ, Phillips RK. Anatomy and function of the anal longitudinal muscle. Br J Surg 1992;79:882-884.

20. Lohsiriwat V. Approach to hemorrhoids. Curr Gastroenterol Rep 2013;15:332.

21. Lestar B, Penninckx F, Kerremans R. The composition of anal basal pressure. An in vivo and in vitro study in man. Int J Colorectal Dis 1989;4:118-122.

22. Hall JF. Modern management of hemorrhoidal disease. Gastroenterol Clin North Am 2013;42:759-772.

23. Schubert MC, Sridhar S, Schade RR, Wexner SD. What every gastroenterologist needs to know about common anorectal disorders. World J Gastroenterol 2009;15:3201-3209.

24. Nivatvongs S-. Hemorrhoids. In Gordon P, Nivatvongs S (editors): Principles and practice of surgery for the colon, rectum and anus, $3^{\text {rd }}$ edition. Informa healthcare, New York London, 2007, pp. 143-166.

25. Fox A, Tietze PH, Ramakrishnan K. Anorectal conditions: hemorrhoids. FP Essent 2014;419:11-19.

26. Peery AF, Sandler RS, Galanko JA, et al. Risk factors for hemorrhoids on screening colonoscopy. PLoS One 2015;10:e139100.

27. Sakakibara R, Tsunoyama K, Hosoi H, et al. Influence of body position on defecation in humans. Low Urin Tract Symptoms 2010;2:16-21.

28. Riss S, Weiser FA, Schwameis K, et al. The prevalence of hemorrhoids in adults. Int J Colorectal Dis 2012;27:215-220.

29. Johanson JF, Sonnenberg A. Constipation is not a risk factor for hemorrhoids: a case-control study of potential etiological agents. Am J Gastroenterol 1994;89:1981-1986.

30. Pigot F, Siproudhis L, Allaert FA. Risk factors associated with hemorrhoidal symptoms in specialized consultation. Gastroenterol Clin Biol 2005;29:1270-1274.

31. Loder PB, Kamm MA, Nicholls RJ, Phillips RK. Haemorrhoids: pathology, pathophysiology and aetiology. Br J Surg 1994;81:946-954.

32. Haas PA, Fox TA Jr. Age-related changes and scar formations of perianal connective tissue. Dis Colon Rectum 1980;23:160-169.

33. Plackett TP, Kwon E, Gagliano RA Jr, Oh RC. Ehlers-Danlos syndrome-hypermobility type and hemorrhoids. Case Rep Surg 2014;2014:171803.

34. Serra R, Gallelli L, Grande R, et al. Hemorrhoids and matrix metalloproteinases: a multicenter study on the predictive role of biomarkers. Surgery 2016;159:487-494.

35. Delcò F, Sonnenberg A. Associations between hemorrhoids and other diagnoses. Dis Colon Rectum 1998;41:1534-1541.

36. Aigner F, Gruber H, Conrad F, et al. Revised morphology and hemodynamics of the anorectal vascular plexus: impact on the course of hemorrhoidal disease. Int J Colorectal Dis 2009;24:105-113.

37. Stankevicius E, Kevelaitis E, Vainorius E, Simonsen U. [Role of nitric oxide and other endothelium-derived factors]. Medicina (Kaunas) 2003;39:333-341.

38. Hancock BD. Internal sphincter and the nature of haemorrhoids. Gut 1977; 18:651-655.

39. Hardy A, Cohen CR. The acute management of haemorrhoids. Ann $R$ Coll Surg Engl 2014;96:508-511.

40. Morgado PJ, Suárez JA, Gómez LG, Morgado PJ Jr. Histoclinical basis for a new classification of hemorrhoidal disease. Dis Colon Rectum 1988;31:474-480.

41. Madoff RD, Fleshman JW; Clinical Practice Committee, American Gastroenterological Association. American Gastroenterological Association technical review on the diagnosis and treatment of hemorrhoids. Gastroenterology 2004;126:1463-1473.

42. Banov L Jr, Knoepp LF Jr, Erdman LH, Alia RT. Management of hemorrhoidal disease. J S C Med Assoc 1985;81:398-401.

43. Garg P. Conservative treatment of hemorrhoids deserves more 
attention in guidelines and clinical practice. Dis Colon Rectum 2018;61:e348.

44. Jacobs DO. Hemorrhoids: what are the options in 2018? Curr Opin Gastroenterol 2018;34:46-49.

45. Tjandra JJ, Tan JJ, Lim JF, Murray-Green C, Kennedy ML, Lubowski DZ. Rectogesic (glyceryl trinitrate $0.2 \%$ ) ointment relieves symptoms of haemorrhoids associated with high resting anal canal pressures. Colorectal Dis 2007;9:457-463.

46. Loder PB, Kamm MA, Nicholls RJ, Phillips RK. 'Reversible chemical sphincterotomy' by local application of glyceryl trinitrate. Br J Surg 1994;81:1386-1389.

47. La Torre F, Nicolai AP. Clinical use of micronized purified flavonoid fraction for treatment of symptoms after hemorrhoidectomy: results of a randomized, controlled, clinical trial. Dis Colon Rectum 2004;47:704-710.

48. Labrid C. Pharmacologic properties of Daflon $500 \mathrm{mg}$. Angiology 1994;45:524-530.

49. Labrid C. A lymphatic function of Daflon $500 \mathrm{mg}$. Int Angiol 1995; 14:36-38.

50. Struckmann JR, Nicolaides AN. Flavonoids. A review of the pharmacology and therapeutic efficacy of Daflon $500 \mathrm{mg}$ in patients with chronic venous insufficiency and related disorders. Angiology 1994;45:419-428.

51. Zagriadskiı EA, Bogomazov AM, Golovko EB. Conservative treatment of hemorrhoids: results of an observational multicenter study. Adv Ther 2018;35:1979-1992.

52. Giannini I, Amato A, Basso L, et al. Flavonoids mixture (diosmin, troxerutin, hesperidin) in the treatment of acute hemorrhoidal disease: a prospective, randomized, triple-blind, controlled trial. Tech Coloproctol 2015;19:339-345.

53. Sneider EB, Maykel JA. Diagnosis and management of symptomatic hemorrhoids. Surg Clin North Am 2010;90:17-32.

54. Klein E, Shapiro R, Ben-Dahan J, Simcha M, Azuri Y, Rosen A. A prospective, randomized, three arm, open label study comparing the safety and efficacy of PP110, a novel treatment for hemorrhoids to preparation $-\mathrm{H}^{\oplus}$ maximum strength cream in the treatment of grade 2-3 hemorrhoids. Mol Cell Ther 2015;3:6.

55. Iyer VS, Shrier I, Gordon PH. Long-term outcome of rubber band ligation for symptomatic primary and recurrent internal hemorrhoids. Dis Colon Rectum 2004;47:1364-1370.

56. Khan AN, Khan NA. A prospective study of outcome from rubber band ligation in third degree haemorrhoids. J Pak Med Assoc 2010;60:952-955.

57. Cocorullo G, Tutino R, Falco N, et al. The non-surgical management for hemorrhoidal disease. A systematic review. G Chir 2017;38:5-14.

58. Beattie GC, Wilson RG, Loudon MA. The contemporary management of haemorrhoids. Colorectal Dis 2002;4:450-454.

59. Haveran LA, Sturrock PR, Sun MY, et al. Simple harmonic scalpel hemorrhoidectomy utilizing local anesthesia combined with intravenous sedation: a safe and rapid alternative to conventional hemorrhoidectomy. Int J Colorectal Dis 2007;22:801-806.

60. Ibrahim S, Tsang C, Lee YL, Eu KW, Seow-Choen F. Prospective, randomized trial comparing pain and complications between diathermy and scissors for closed hemorrhoidectomy. Dis Colon Rectum 1998;41:1418-1420.

61. Seow-Choen F, Ho YH, Ang HG, Goh HS. Prospective, randomized trial comparing pain and clinical function after conventional scissors excision/ligation vs. diathermy excision without ligation for symptomatic prolapsed hemorrhoids. Dis Colon Rectum 1992;35:1165-1169.

62. Chen JS, You JF. Current status of surgical treatment for hemorrhoids-systematic review and meta-analysis. Chang Gung Med J 2010;33:488-500.

63. Ferguson JA, Mazier WP, Ganchrow MI, Friend WG. The closed technique of hemorrhoidectomy. Surgery 1971;70:480-484.

64. Sutherland LM, Burchard AK, Matsuda K, et al. A systematic review of stapled hemorrhoidectomy. Arch Surg 2002;137:13951406; discussion 1407.

65. Tjandra JJ, Chan MK. Systematic review on the procedure for prolapse and hemorrhoids (stapled hemorrhoidopexy). Dis Colon Rectum 2007;50:878-892.

66. Morinaga K, Hasuda K, Ikeda T. A novel therapy for internal hemorrhoids: ligation of the hemorrhoidal artery with a newly devised instrument (Moricorn) in conjunction with a Doppler flowmeter. Am J Gastroenterol 1995;90:610-613.

67. Brown SR. Haemorrhoids: an update on management. Ther $A d v$ Chronic Dis 2017;8:141-147.

68. Faucheron JL, Gangner Y. Doppler-guided hemorrhoidal artery ligation for the treatment of symptomatic hemorrhoids: early and three-year follow-up results in 100 consecutive patients. Dis Colon Rectum 2008;51:945-949. 